\begin{tabular}{|c|l|}
\hline Title & Molecular-based light-activated thy ristor \\
\hline Author(s) & Iimori, Toshifumi; Ohta, Nobuhiro; Naito, Toshio \\
\hline Citation & $\begin{array}{l}\text { A pplied Physics Letters, 90(26), 262103 } \\
\text { https://doi.org/10.1063/2.2749845 }\end{array}$ \\
\hline Issue Date & 2007-06-25 \\
\hline Doc URL & http://hdl.handle.net/2115/28277 \\
\hline Rights & Copyright $\odot 2007$ A merican Institute of Physics \\
\hline Type & article \\
\hline File Information & APL90-26.pdf \\
\hline
\end{tabular}

Instructions for use 


\title{
Molecular-based light-activated thyristor
}

\author{
Toshifumi limori and Nobuhiro Ohta ${ }^{a}$ \\ Research Institute for Electronic Science, Hokkaido University, Sapporo 060-0812, Japan \\ Toshio Naito \\ Creative Research Initiative "Sousei," Hokkaido University, Sapporo 001-0021, Japan and Division \\ of Chemistry, Graduate School of Science, Hokkaido University, Sapporo 060-0811, Japan
}

(Received 9 April 2007; accepted 24 May 2007; published online 25 June 2007)

\begin{abstract}
A photoinduced electrical conductivity switching is observed in the molecular conductor of $\alpha$-[bis(ethylenedithio)tetrathiafulvalene $]_{2} \mathrm{I}_{3}$ at different temperatures with different irradiation light intensities. The threshold voltage for the differential-negative-resistance effect appearing in the current-voltage characteristic curve decreases and increases, respectively, as the light intensity is increased and as the temperature decreases below the insulator-metal phase transition. The potential application of molecular conductor in bidirectional light-activated thyristor devices is demonstrated. (C) 2007 American Institute of Physics. [DOI: 10.1063/1.2749845]
\end{abstract}

Molecular-based materials have been discussed as candidates for advanced functional materials. ${ }^{1-4}$ Organic chargetransfer (CT) complexes have, in particular, received intense attention as prospective materials ${ }^{5}$ since high electrical conductivity in the CT compound between perylene and bromide was discovered by Akamatu et al. in $1954 .{ }^{6}$ So far a great number of organic CT complexes have been developed and are known to show not only the electrical conductivity but also versatile, intriguing, and technically useful properties such as superconductivity, ${ }^{7,8}$ magnetism, ${ }^{9-11}$ ferromagnetism, ${ }^{12}$ optical nonlinearity, ${ }^{13}$ and ion conductivity. ${ }^{14}$ In the current-voltage $(I-V)$ characteristics, field-induced switching effects have been discovered recently in several molecular conductors. ${ }^{5,15-17}$ The fieldinduced switching effect can, in general, be attributed to the differential-negative-resistance (DNR) effect in the $I-V$ characteristic curve of materials. ${ }^{18,19}$ The structural change upon the switching has been investigated for a few molecular conductors using $\mathrm{x}$-ray crystallographic methods. ${ }^{16,20}$ These studies have shown that the relaxation of charge ordering or disproportion of electrons takes place in a high conductivity (HC) state in the materials showing the DNR effect.

The switching effect originating from the DNR effect in materials is an important practical functionality for use in electronic devices such as a memory or a semiconductor controlled rectifier (SCR). SCR or the so-called thyristor device, which is used in many industrial products such as inverters and power converters, has a four-layer $p-n-p-n$ structure of inorganic semiconductor. ${ }^{19}$ As understood from the $p-n$ junction structure, the thyristor devices exhibit a switching characteristic in only one bias direction and a blocking characteristic in the opposite direction. ${ }^{19}$ Components that show bidirectional switching characteristics can be obtained by assembling two thyristor devices having the opposite switching directions into a single unit. On the other hand, the DNR effect in molecular conductors is intrinsically bidirectional because the component showing the DNR effect is composed of a single compound. This characteristic enables us to construct the bidirectional switching devices having a simpler structure than those using the inorganic semiconductors.

\footnotetext{
a) Author to whom correspondence should be addressed; electronic mail: nohta@es.hokudai.ac.jp
}

There are several methods to trigger the switching effect in conventional thyristor devices. ${ }^{19}$ When the magnitude of the voltage between the terminals of the device exceeds a certain threshold value, the switching to a $\mathrm{HC}$ state can take place. This voltage triggering is the most important method of the switching of thyristor devices. The threshold voltage to induce the switching can be decreased with an increase of the current flow through a gate electrode on the device. Light irradiation can also change the threshold voltage, and the threshold voltage decreases with an increase of the light intensity. Using the light triggering method, we can electrically isolate the triggering circuit from the switching circuit composed of the $p-n-p-n$ structure. Owing to this technical advantage, the light triggering method is widely utilized in high-current switching units. Further, this triggering method is required to construct optoelectronic devices such as optical switches, which are utilized as building blocks in an optical information processing. The thyristor devices employing the light triggering method are often called the light-activated thyristor.

The investigation of the light-activated thyristorlike function has been still quite rare in molecular conductors. Observation of the DNR effect in the $I-V$ characteristic curve in the light-irradiated polycrystalline thin film of copper tetracyanoquinodimethane has been reported by Potember et al. $^{21}$ However, a basic characterization such as the light intensity dependence and temperature dependence of the DNR effect in the $I-V$ characteristic curve has not been performed. Moreover, characterization of a single crystal of molecular conductors has not been reported so far. Here, we report that the circuit including a single crystal of a molecular conductor acts as a conventional light-activated thyristor, and the DNR effect in the $I-V$ characteristic curve can be controlled by the light intensity and operation temperature.

The CT complexes based on bis(ethylenedithio)tetrathiafulvalene (BEDT-TTF) donor molecule are a family of compounds that show rich low-dimensional physics such as metal-insulator $(M-I)$ phase transition and superconductivity. ${ }^{22} \alpha$-(BEDT-TTF $)_{2} \mathrm{I}_{3}$ has an arrangement of a donor layer classified as $\alpha$ type and has a layered structure composed of a conducting BEDT-TTF layer and an insulating $\mathrm{I}_{3}^{-}$layer. In the unit cell, there are four BEDT-TTF molecules and two $\mathrm{I}_{3}^{-}$molecules, and consequently this crys- 


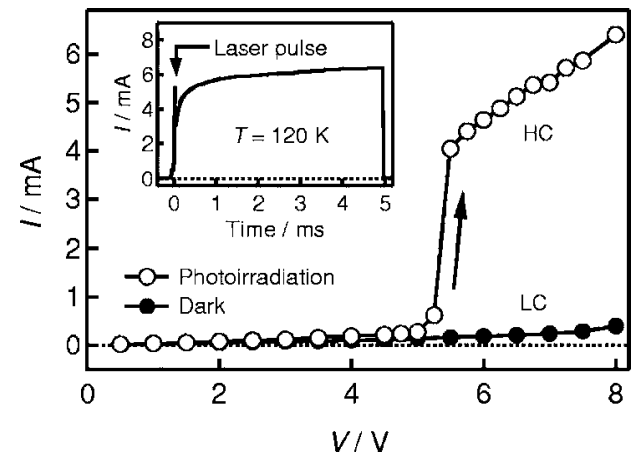

FIG. 1. Plots of the current $(I)$ vs the applied voltage $(V)$ in the circuit including the $\alpha$-(BEDT-TTF $)_{2} \mathrm{I}_{3}$ crystal at a temperature $(T)$ of $120 \mathrm{~K}$. The curves were measured with photoirradiation of $3.4 \times 10^{-5} \mathrm{~J} /$ pulse and without photoirradiation. Arrow indicates the direction of the current change. (Inset) Temporal profile of the current observed with the photoirradiation using the pulsed voltage of $8 \mathrm{~V} \cdot R_{L}=1 \mathrm{k} \Omega$.

tal has $3 / 4$-filled band. $\alpha$-(BEDT-TTF $)_{2} \mathrm{I}_{3}$ is a metal at room temperature and shows the $M-I$ transition at $T_{M-I}=135 \mathrm{~K}^{23}$ Spectroscopic and theoretical investigations have shown that a charge-ordering state is realized in the insulator phase. ${ }^{24,25}$

A single crystal of $\alpha$-(BEDT-TTF $)_{2} \mathrm{I}_{3}$ was prepared by an electrocrystallization method. ${ }^{23}$ The dimension of typical samples was $1 \times 0.5 \times 0.05 \mathrm{~mm}^{3}$. The measurement method used in this work is essentially the same as that reported elsewhere. ${ }^{26}$ The sample was placed in a cryostat (a temperature-controlled He buffer gas) using two gold wires separated by a distance $(d)$ of approximately $0.2 \mathrm{~mm}$, which served as electrodes. We used a pulsed voltage as a bias voltage $(V)$, and a temporal profile of the current was monitored using a digital oscilloscope connected to the circuit in series with the sample. As a light source in photoirradiation experiments, the second harmonic $(532 \mathrm{~nm})$ of output from a $\mathrm{Nd}$ doped yttrium aluminum garnet laser (QuantaRay, DCR$11,10 \mathrm{~ns}$ pulse width, and repetition rate of $8.3 \mathrm{~Hz}$ ) was used. The laser light was applied to the sample between the two electrodes in synchronization with the pulsed voltage. We inserted a load resistance $\left(R_{L}\right)$ in series into the circuit to avoid damage to the sample by excessive current loading.

The $I-V$ characteristic curve of the $\alpha$-(BEDT-TTF $)_{2} \mathrm{I}_{3}$ crystal was measured in the insulator phase at various temperatures $(T)$ near the $T_{M-I}$. When the sample was photoexcited by the laser pulse, the photocurrent was observed, and the magnitude of the photocurrent became smaller with the lifetime shorter than a few microseconds, as far as the applied voltage was not so strong. ${ }^{27}$ The pulse width of the applied voltage used in the present study was $5 \mathrm{~ms}$, which was much longer than the photocurrent lifetime, and so the transient photocurrent did not contribute to the current observed at the end of the pulsed voltage. When the pulse height of the applied voltage was large enough, however, the current which was initially induced by photoirradiation could be retained as far as the voltage was applied (see inset of Fig. 1). This observation is ascribed to the switching effect in the electrical conductivity. Figure 1 shows the $I-V$ characteristic curve observed at $T=120 \mathrm{~K}$ with and without photoirradiation. These curves were measured by scanning $V$ in the positive direction; the current was measured with an increase of the applied voltage. The current $(I)$ observed at the end of the applied pulsed voltage, i.e., $I$ at approximately $4.9 \mathrm{~ms}$ after photoexcitation was used for the $I-V$ characteristic curve shown hereafter. Without photoirradiation, the sample was in Downloaded 24 Sep 2007 to 133.87.26.100. Redistribution subject
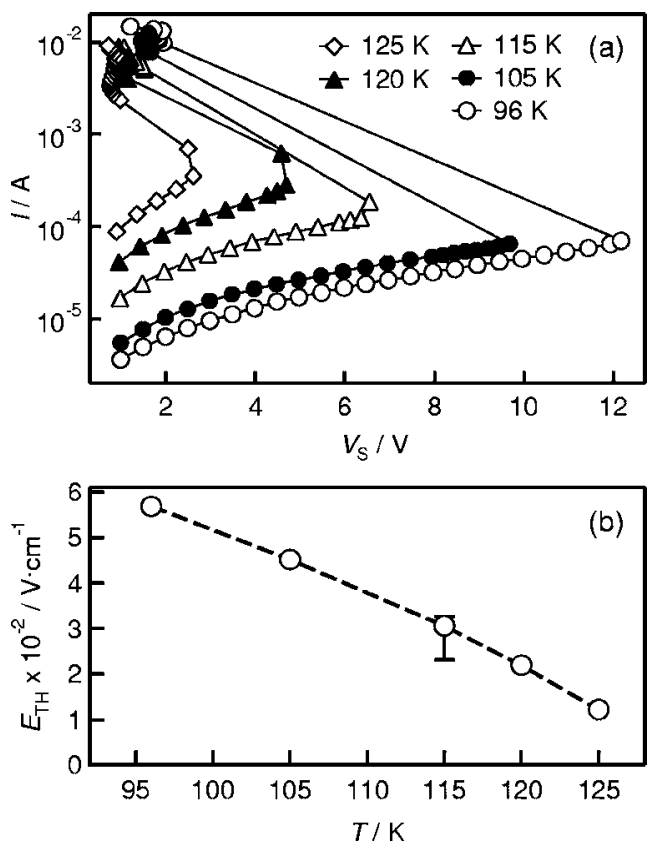

FIG. 2. (a) Plots of the current $(I)$ vs the voltage applied to the sample $\left(V_{S}\right)$ observed with the photoirradiation at different temperatures. Photoirradiation intensity was fixed to $3.4 \times 10^{-5} \mathrm{~J} /$ pulse. (b) Plot of the threshold electric field $\left(E_{\mathrm{th}}\right)$ at which the switching effect in the $I-V$ characteristic curves appeared versus temperature. Error bar is put on the data at $T=115 \mathrm{~K}$.

the low conductivity (LC) state that can be assigned to the insulator phase of the crystal. The $I-V$ characteristic curve observed with photoirradiation having intensity of 3.4 $\times 10^{-5} \mathrm{~J} /$ pulse showed the discontinuous increase at approximately $5 \mathrm{~V}$. The inset of Fig. 1 shows the temporal profile of the current observed with the photoirradiation and the pulsed voltage having the height of $8.0 \mathrm{~V}$.

At the temperatures investigated in the present study, the $R_{L}$ value, i.e., $1 \mathrm{k} \Omega$, was much smaller than the dark resistance of the sample. Thus, we can consider that most of the voltage applied to the whole circuit (i.e., $V$ ) was applied to the sample. The voltage drop at the sample (denoted by $V_{S}$ ) can be calculated from the relation $V_{S}=V-\left(R_{0}+R_{L}\right) I$, where $R_{0}$ represents circuit resistance other than that of the sample. Plots of the current versus $V_{S}$ are shown in Fig. 2(a), in which the experimental results at temperatures between 96 and $125 \mathrm{~K}$ are shown. For all the measurements, the photoirradiation intensity of $3.4 \times 10^{-5} \mathrm{~J} /$ pulse (matching approximately $4 \mathrm{MW} / \mathrm{cm}^{2}$ ) was used. When the $V_{S}$ exceeds certain threshold values $\left(V_{\mathrm{th}}\right)$, the electrical conductivity switches from the LC state to the HC state along the load line of the circuit. This result indicates that the DNR effect is observed in the photoirradiated crystal.

The $I-V_{S}$ characteristic curves shown in Fig. 2(a) clearly demonstrate that the $V_{\text {th }}$ strongly depends on temperature. Figure 2(b) shows the plot of the threshold electric field $E_{\mathrm{th}}$, which is calculated from the $V_{\text {th }}$ divided by $d$, as a function of the sample temperature. The $E_{\mathrm{th}}$ showed almost linear dependence on temperature with a negative slope. In the temperature range used in the present work, we can vary $E_{\text {th }}$ from approximately $1 \times 10^{2}$ to approximately $6 \times 10^{2} \mathrm{~V} / \mathrm{cm}$. These values are rather small in comparison with those of other organic materials that show the field-induced DNR effect. ${ }^{15}$ This feature may be technically advantageous since the pulsed voltage with a height lower than $10 \mathrm{~V}$ can induce the DNR effect, as shown in Figs. 1 and 2, and this range of to AIP license or copyright, see http://apl.aip.org/apl/copyright.jsp 

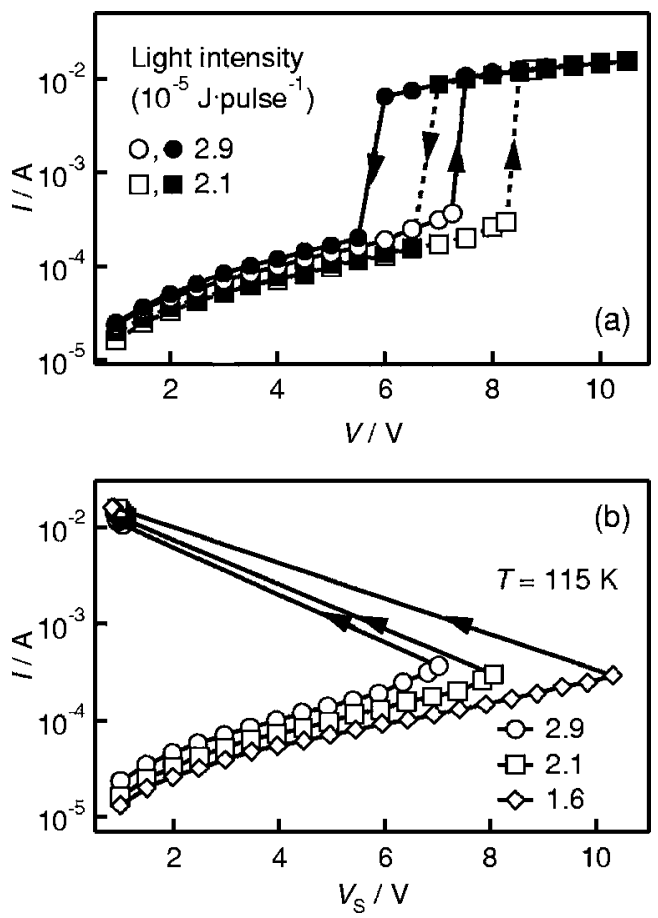

FIG. 3. Electrical characterization of the circuit at different photoirradiation intensities. $T=115 \mathrm{~K} . R_{L}=510 \Omega$. (a) The $I-V$ characteristic curves obtained with the photoirradiation at the intensities of $2.1 \times 10^{-5} \mathrm{~J} /$ pulse (dotted line) and $2.9 \times 10^{-5} \mathrm{~J} /$ pulse (solid line). The voltage was scanned both in the positive direction (open mark) and in the negative direction (closed mark). Arrows indicate the direction of the current change. (b) The $I-V_{S}$ characteristic curves calculated from the $I-V$ data observed with the photoirradiation. The intensities of the photoirradiation were taken as $1.6 \times 10^{-5}, 2.1 \times 10^{-5}$, and $2.9 \times 10^{-5} \mathrm{~J} /$ pulse.

the voltage height is compatible with those used in the conventional electrical circuits. The feature in the LC-HC switching cycle was reproducible. Actually, the value of $E_{\text {th }}$ slightly decreased after the repeated photoirradiation; the $E_{\mathrm{th}}$ of a certain sample varied from $2.4 \times 10^{2}$ to 2.3 $\times 10^{2} \mathrm{~V} / \mathrm{cm}$ after the experiment lasting $6 \mathrm{~h}$ at the typical irradiation intensity of $3 \times 10^{-5} \mathrm{~J} /$ pulse.

As noted in the introduction, the pivotal characteristic in the operation of light-activated thyristor devices is the controllability of the $E_{\text {th }}$ by light intensity. Then we investigated the dependence of $E_{\text {th }}$ on the light intensity. Figure 3(a) shows the $I-V$ characteristic curves obtained at different light intensities at a fixed temperature $(T=115 \mathrm{~K})$. These curves were obtained with the voltage height scanning in the positive and negative directions. With the photoirradiation at $2.1 \times 10^{-5} \mathrm{~J} /$ pulse, the LC-to-HC switching took place at $V$ $\approx 8 \mathrm{~V}$, and the switching back to the LC state was observed at a lower $V$ in the negative voltage scan. Thus, the electric circuit including the $\alpha$-(BEDT-TTF $)_{2} \mathrm{I}_{3}$ crystal can show the bistability in a certain range of $V$. The $I-V_{S}$ characteristic curves obtained at the different light intensities are shown in Fig. 3(b). For clarity, only the curves obtained by scanning the voltage height in the positive direction are shown. As the light intensity was increased, the $V_{\text {th }}$ for the DNR effect obviously decreased. With the photoirradiation intensity used in this study, photoinduced effects which damage the sample such as a laser abrasion did not appear.

In the insulator phase, i.e., the LC state, the current at the end of the applied pulsed voltage was in the same order of magnitude with the dark current even with photoirradiation (see Fig. 1). However, the photoirradiation in the presence of electric fields generates the transient photocurrent. We have recently reported that the transient photocurrent at temperatures near the $T_{M-I}$ can be ascribed to the photoinduced insulator-metal $(I-M)$ phase transition. ${ }^{27}$ Thus, it is probable that the photoinduced $I-M$ phase transition plays an important role in the triggering of the DNR effect in the photoirradiated crystal. The DNR effect often accompanies periodic current oscillations or instabilities in the circuit. In this study, however, such a current oscillation was not observed.

In summary, we have demonstrated that the $\alpha$-(BEDT-TTF $)_{2} \mathrm{I}_{3}$ crystal can show the current bistability and the DNR effect with photoirradiation. The dependences of the $I-V_{S}$ curve on temperature and on light intensity were characterized. These results show the potential application of molecular conductor in molecular based materials for bidirectional light-activated thyristor devices at temperatures below the $T_{M-I}$.

This work was supported by a Grant-in-Aid for Scientific Research (Grant No. 15205001) from the Ministry of Education, Culture, Sports, Science and Technology in Japan.

${ }^{1}$ R. M. Metzger, Chem. Rec. 4, 291 (2004).

${ }^{2}$ G. Saito and Y. Yoshida, Bull. Chem. Soc. Jpn. 80, 1 (2007).

${ }^{3}$ T. Naito, T. Inabe, H. Niimi, and K. Asakura, Adv. Mater. (Weinheim, Ger.) 16, 1786 (2004).

${ }^{4}$ T. W. Kelley, P. F. Baude, C. Gerlach, D. E. Ender, D. Muyres, M. A. Haase, D. E. Vogel, and S. D. Theiss, Chem. Mater. 16, 4413 (2004).

${ }^{5}$ S. Horiuchi, T. Hasegawa, and Y. Tokura, J. Phys. Soc. Jpn. 75, 051016 (2006).

${ }^{6}$ H. Akamatu, H. Inokuchi, and Y. Matsunaga, Nature (London) 173, 168 (1954).

${ }^{7}$ J. M. Williams, A. J. Schultz, U. Geiser, K. D. Carlson, A. M. Kini, H. H. Wang, W.-K. Kwok, M.-H. Whangbo, and J. E. Schirber, Science 252, 1501 (1991)

${ }^{8}$ H. Kobayashi, H. Cui, and A. Kobayashi, Chem. Rev. (Washington, D.C.) 104, 5265 (2004).

${ }^{9}$ J. S. Miller and A. J. Epstein, Angew. Chem., Int. Ed. Engl. 33, 385 (1994).

${ }^{10}$ E. Coronado and P. Day, Chem. Rev. (Washington, D.C.) 104, 5419 (2004).

${ }^{11}$ W. Fujita and K. Awaga, Science 286, 261 (1999).

${ }^{12}$ E. Coronado, J. R. Galan-Mascaros, C. J. Gomez-Garcia, and V. Laukhin, Nature (London) 408, 447 (2000).

${ }^{13}$ P. G. Huggard, W. Blau, and D. Schweitzer, Appl. Phys. Lett. 51, 2183 (1987).

${ }^{14}$ T. Nakamura, T. Akutagawa, K. Honda, A. E. Underhill, A. T. Coomber, and R. H. Friend, Nature (London) 394, 159 (1998).

${ }^{15}$ K. Okamoto, T. Tanaka, W. Fujita, K. Awaga, and T. Inabe, Angew. Chem., Int. Ed. 45, 4516 (2006).

${ }^{16}$ M. M. Matsushita and T. Sugawara, J. Am. Chem. Soc. 127, 12450 (2005).

${ }^{17}$ Y. Iwasa, T. Koda, S. Koshihara, Y. Tokura, N. Iwasawa, and G. Saito, Phys. Rev. B 39, 10441 (1989).

${ }^{18}$ B. K. Ridley, Proc. Phys. Soc. London 82, 954 (1963).

${ }^{19}$ S. M. Sze, Physics of Semiconductor Devices (Wiley, New York, 1969).

${ }^{20}$ F. Sawano, I. Terasaki, H. Mori, T. Mori, M. Watanabe, N. Ikeda, Y. Nogami, and Y. Noda, Nature (London) 437, 522 (2005).

${ }^{21}$ R. S. Potember, T. O. Poehler, and R. C. Benson, Appl. Phys. Lett. 41, 548 (1982).

${ }^{22}$ R. P. Shibaeva and E. B. Yagubskii, Chem. Rev. (Washington, D.C.) 104, 5347 (2004).

${ }^{23}$ K. Bender, I. Hennig, D. Schweitzer, K. Dietz, H. Endres, and H. J. Keller, Mol. Cryst. Liq. Cryst. 108, 359 (1984).

${ }^{24}$ T. Takahashi, Y. Nogami, and K. Yakushi, J. Phys. Soc. Jpn. 75, 051008 (2006).

${ }^{25}$ H. Seo, C. Hotta, and H. Fukuyama, Chem. Rev. (Washington, D.C.) 104, 5005 (2004).

${ }^{26}$ T. Iimori, T. Naito, and N. Ohta, J. Am. Chem. Soc. 129, 3486 (2007).

${ }^{27}$ T. Iimori, T. Naito, and N. Ohta, Chem. Lett. 36, 536 (2007). 